\title{
Современные возможности пренатальной и постнатальной диагностики синдрома Фринса: собственные наблюдения и обзор литературы
}

\author{
Румянцева Н.В., Хурс О.М., Новикова И.В., Венчикова Н.А., Зобикова О.Л. \\ ГУ «Республиканский научно-практический центр «Мать и дитя» Министерства здравоохранения Республики Беларусь \\ Минск, Республика Беларусь
}

Синдром Фринса (СФ, ОМІМ\#194050) - редкое аутосомно-рецессивное заболевание с неустановленным генетическим дефектом и высокой летальностью, манифестирует характерным комплексом врожденных пороков развития (ВПР), включающим диафрагмальную грыжу (ДГ). Представлен обзор литературы по фенотипическим признакам и диагностическим критериям синдрома, приведены клинико-генеалогические данные опубликованных семейных наблюдений, ультразвуковые (УЗ) и патоморфологические характеристики плодов с СФ.

Цель исследования: проанализировать пренатальные УЗ, клинические и патоморфологические характеристики 12 пациентов с СФ, выявленных в Беларуси за период 2009-2018 гг. и представить результаты ретроспективного анализа репродукции семей. Материалы и методы. Проведено медико-генетическое консультирование 10 семей. Все беременные прошли комбинированный пренатальный скрининг. Выполнены пре- и постнатальный анализы кариотипов (GTG-banding), патоморфологическое исследование 10 абортированных плодов и 2 умерших младенцев.

Результаты и обсуждение. Фенотипические проявления у всех пациентов соответствовали диагностическим критериям СФ. Среди пренатальных УЗ признаков в 1 триместре при 5 беременностях установлено расширение воротникового пространства; во 2 триместре у 11 плодов выявлена ДГ, у одного - омфалоцеле, в 4 случаях - другие аномалии развития. Грубые черты лица отмечены у живорожденных и плодов. Гипоплазия легких установлена у всех пациентов, редкие аномалии (легочной секвестр, кистозно-аденоматозный порок, аплазия доли) - в 5 случаях. Характерные дефекты терминальных фаланг и/или ногтевых пластинок отмечены в 11 наблюдениях, у 3 сибсов выявлены редкие пороки - полидактилия кистей, эктродактилия стоп. Среди ассоциированных аномалий развития наблюдались расщелина неба, аринэнцефалия, гидроцефалия, комбинированный порок сердца, омфалоцеле, дивертикул Меккеля, аномалии почек, двурогая матка и гипоспадия. В семейном наблюдении у 3 сибсов отмечено высокое сходство фенотипических проявлений. Анализ исходов беременностей в 10 семьях свидетельствует об отягощенном акушерском анамнезе. Из 29 беременностей в 12 случаях потомство имело СФ, 4 завершились спонтанным абортом, 11 - рождением здоровых детей, при 2 текущих беременностях ВПР у плодов не выявлены.

Заключение. Клинический диагноз СФ устанавливается при соответствии комплекса ВПР принятым диагностическим критериям после исключения хромосомного дисбаланса. При обнаружении у плода ДГ - ведущего диагностического признака, следует проводить целенаправленный поиск ассоциированных аномалий. Полидактилия и эктродактилия могут рассматриваться в качестве элементов фенотипического спектра СФ.

Ключевые слова: синдром Фринса, аутосомно-рецессивный тип наследования, диафрагмальная грыжа, гипоплазия/аплазия терминальных фаланг и ногтевых пластинок, полидактилия, эктродактилия, пренатальная диагностика.

Для цитирования: Румянцева Н.В., Хурс О.М., Новикова И.В., Венчикова Н.А., Зобикова О.Л. Современные возможности пренатальной и постнатальной диагностики синдрома Фринса: собственные наблюдения и обзор литературы. Медицинская генетика 2020; 19(1): 13-23 DOI: $10.25557 / 2073-7998.2020 .01 .13-23$

Автор для корреспонденции: Румянцева Наталья Владимировна; e-mail: rumiantseva@inbox.ru

Финансирование отсутствует.

Конфликт интересов. Авторы декларируют отсутствие конфликта интересов.

Поступила: 15.01.2020

\section{The possibilities of prenatal and postnatal Fryns syndrome diagnostics: patients presentations and overview}

Rumiantseva N.V., Khurs O.M., Novikova I.V., Venchikova N.A., Zobikova O.L.

Republican Scientific Practical Centre «Mother and Child», Ministry of Health, Belarus

Minsk, Belarus

Fryns syndrome (FS, OMIM\#194050) is a rare lethal autosomal recessive disorder with unknown genetic defect, manifested the distinct complex of the multiply congenital abnormalities (MCA), including diaphragmatic hernia. There was made the world literature overview for the FS phenotypic features, clinical and genealogical data of the published familial cases, ultrasound and morphological characteristics of affected fetuses. 
Aim. Analysis of the clinical, morphological and prenatal ultrasound data of 12 FS cases, detected in Belarus during $2009-2018$ years, and families' reproduction results.

Material and methods. Genetic counseling of 10 families with affected outcome was performed. All the pregnants underwent the combined (biochemical and ultrasound) screening. Prenatal and postnatal cytogenetical studies (GTG-banding) and the morphological investigations of the 10 aborted fetuses and 2 deceased infants were fulfilled.

Results and Discussion. All the patients presented typical FS phenotypic features correlated with the diagnostic criteria. The spectrum of prenatal findings diagnosed at 1-st trimester included nuchal skin enlargement/cystic hygroma (5 cases), at the 2-ed trimester - diaphragmatic hernia (11 cases), omphalocele (1), other malformations (4 fetuses). The facial dysmorphisms (coarse face) were detected in all the patients. Lungs hypoplasia was found in all cases, 5 patients additionally dysplayed other abnormalities: pulmonary sequestration, cystic-adenomatoid malformation, lobular aplasia. Characteristic phalanges and nails hypoplasia or aplasia were registered in 11 cases, 3 sibs presented rare malformations - polydactyly (hands) and ectrodactyly (feet). Associated malformations spectrum included cleft palate, brain abnormalities (ventricular dilatation, arhinencephaly), cardiac defects, omphalocele, Meckel diverticule, hydronephroses, renal cysts, bicornuate uterus and hypospadia. The familial case with 3 affected sibs demonstrated a similarity in main diagnostic criteria. The couples reproduction analysis illulustrated a serious failure. Outcome of 29 pregnancies were as follows: 12 - FS cases, 4 - miscarries at 1-st trimester, 11 - healthy offspring and 2 cases - pregnancies are going on currently (both fetuses without malformations).

Conclusion. Clinical diagnosis of FS may be noted in patients (either fetuses or newborns), which display the conjunction of typical MCA pattern with a normal karyotype. Additional investigations must be used in order to exclude the associated abnormalities if diaphragmatic hernia has been detected in fetus. In our opinion polydactyly and ectrodactyly may be added to the FS phenotypic spectrum.

Key words: Fryns syndrome, autosomal recessive inheritance, diaphragmatic hernia, phalanges and nails hypoplasia or aplasia, polydactyly, ectrodactyly, prenatal diagnostics.

For citation: Rumiantsava N.V., Khurs O.M., Novikova I.V., Venchikova N.A., Zobikova O.L. The possibilities of prenatal and postnatal Fryns syndrome diagnostics: patients presentations and overview. Medical genetics 2020; 19(1): 13-23. [In Rus].

DOI: $10.25557 / 2073-7998.2020 .01 .13-23$

Corresponding author. Rumiantseva Natalia, e-mail: rumiantseva@inbox.ru

Funding is absent.

Conflict of interest. The authors declare no conflict of interest.

Accepted: 15.01 .2020

\section{Введение}

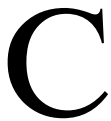
индром Фринса (СФ, ОМІМ\#194050) описан Fryns J. и соавт. в 1979 г. у 2 мертворожденных сибсов, имевших грубые черты лица, помутнение роговицы, расщелину неба, диафрагмальную грыжу (ДГ), гипоплазию легких, гипоплазию терминальных фаланг и ногтевых пластинок [1]. Впоследствии, на основе анализа фенотипа 8 пациентов были выделены ведущие диагностические признаки [2].

Опубликованы описания около 120 случаев живорожденных и плодов с СФ. Рождение больного потомства в кровнородственном браке [3, 4], наблюдения разнополых сибсов с СФ у здоровых родителей [2-8] свидетельствуют об аутосомно-рецессивном типе наследования. Генетический дефект, приводящий к возникновению заболевания, не идентифицирован. Описаны пациенты с фенотипическими проявлениями СФ и мутациями в гене $P I G N[9,10]$.

Клинический диагноз устанавливается при наличии характерного комплекса врожденных аномалий развития после исключения хромосомного дисбаланса. Спектр и частота фенотипических признаков детально представлены в обзоре Slavotinek A. [11].
Черепно-лицевые дисморфии (ЧЛД) формируют характерный паттерн признаков, который описан как «грубые черты лица» («coarse face»): низкопосаженные дисморфичные ушные раковины $(55,8 \%)$, гипертелоризм $(36,5 \%)$, широкое переносье $(38,5 \%)$, толстый кончик носа, вывернутые ноздри $(26,9 \%)$, макростомия $(38,5 \%)$, форма верхней губы в виде «палатки» («tented» lip), микроретрогения (36,5\%).

Аномалии диафрагмы (частота 96\%) могут быть представлены вариантами левосторонней $(53,8 \%$, тип Bochdalek), билатеральной $(19,2 \%)$ или правосторонней $(11,5 \%)$ ДГ, реже гипо/аплазией и эвентрацией диафрагмы.

Патология легких включает гипоплазию $(65,4 \%)$ и нарушение лобуляции $(9,6 \%)$.

Пороки конечностей представлены гипоплазией/ аплазией терминальных фаланг пальцев кистей/стоп $(59,6 \%)$, аплазией/гипоплазией ногтевых пластинок $(59,6 \%)$. Отмечаются широкие $(13,5 \%)$ первые пальцы кистей с проксимальной позицией $(15,4 \%)$, камптодактилия $(11,5 \%)$; описаны косолапость $(19,2 \%)$, стопа-качалка $(3,8 \%)$. 
Ассоциированные аномалии:

- помутнение роговицы $(17,3 \%)$, реже микрофтальм (5,8\%);

- расщелина неба (50\%), расщелина губы (25\%) и их сочетание;

- структурные аномалии мозга (88\%): гидроцефалия/ расширение желудочков (23\%), агенезия мозолистого тела $(13,5 \%)$, нейрональные/церебеллярные гетеротопии $(13,5 \%)$, порок Дэнди-Уокера (9,6\%), полимикрогирия, гипоплазия мозжечка $(3,8 \%)$, отсутствие обонятельных луковиц/трактов $(3,8 \%)$, ариненцефалия $(1,9 \%)$;

- сердечно-сосудистая система (ССС): септальные дефекты (ДМЖП - 40,4\%, ДМПП - 13,5\%), аортальные $(13,5 \%)$ и конотрункальные пороки;

- желудочно-кишечный тракт (ЖКТ): нарушения поворота кишечника $(15,4 \%)$, омфалоцеле $(9,6 \%)$, дивертикул Меккеля $(9,6 \%)$, аноректальные аномалии $(9,6 \%)$ и полиспления $(9,6 \%)$;

- пороки мочеполовой системы: кисты и дисплазия почек $(26,9 \%$ и $11,5 \%)$, гидроуретер/гидронефроз $(17,3 \%$ и 5,8\%), гипоплазия мочевого пузыря, фистула уретры, двурогая матка $(11,5 \%)$, двойственное строение гениталий $(5,8 \%)$, гипоспадия $(1,9 \%)$;

- прогрессирующее многоводие со 2 триместра беременности $(55,8 \%)$.

B 2005 г. Lin A. и соавт. провели анализ наибольшей когорты пациентов с СФ (109 опубликованных и 3 собственных наблюдения) и предложили обновленную формулировку диагностических критериев синдрома: (1) аномалии диафрагмы, (2) характерные ЧЛД, (3) гипоплазия/аплазия терминальных фаланг и/или ногтевых пластинок кистей и стоп, (4) гипоплазия легких, (5) ассоциированные аномалии, (6) наличие больных сибсов [12]. Используя 6 диагностических критериев, были выделены 3 категории пациентов с СФ: (1) имеют 4 и более из 6 критериев, (2) имеют 3 признака, (3) атипичный СФ - с отсутствием ДГ $(9,8 \%$ случаев) либо наличием нехарактерных пороков развития (полидактилия, эктродактилия, птеригии и др.) [12].

Семейный анамнез с наличием больных сибсов имеет важное значение для установления нозологического диагноза. Опубликовано 16 семейных наблюдений СФ с 36 пораженными сибсами (живорожденные и умершие младенцы, плоды). В 13 семьях было по 2 больных [5-8, 13-17], в том числе 3 пары монозиготных близнецов, 2 из которых родились в кровнородственных браках [15-17], 2 семьи имели по 3 пораженных сибса (младенец и 2 плода) [14, 18], в одной семье родилось 4 детей с ДГ и ассоциированными аномалиями [19].

Заболевание характеризуется высокой летальностью. По данным литературы абсолютное большин- ство беременностей плодом с СФ завершилось летальным исходом (мертворождение и дети, умершие в неонатальном периоде). В то же время, известны наблюдения 11 детей с фенотипом СФ в возрасте от 1 до 15 лет, имеющих тяжелую задержку психоречевого развития, неврологические расстройства, судорожный синдром [20].

Поскольку генетический дефект не установлен, эффективность профилактики рождения потомства с СФ определяется возможностями пренатальной УЗ диагностики [21]. Однако, в мировой практике случаи диагностики СФ с цитогенетическим и патоморфологическим исследованиями плода немногочисленны.

Цель исследования: проанализировать пренатальные УЗ, клинические и патоморфологические характеристики 12 пациентов с СФ, выявленных в Беларуси за период 2009-2018 гг., и представить результаты ретроспективного анализа репродукции семей.

\section{Материалы и методы}

Группу исследования составили 10 семей, имеющих потомство с комплексом МВПР, включающим ДГ (9 изолированных и одно семейное наблюдение с 3 пораженными сибсами).

Все беременные прошли комбинированный пренатальный скрининг. УЗ исследования проводились на сканерах Sonoline Versa Plus (Siemens) и Voluson 730 Expert (GE) с использованием трансвагинального и трансабдоминального датчиков.

Пренатальный анализ кариотипов выполнен на препаратах клеток культур ворсин хориона и амниоцитов, постнатальный - лимфоцитов периферической крови с использованием метода дифференциальной (GTG-banding) окраски хромосом.

Проведено патоморфологическое исследование 10 плодов, абортированных по медицинским показаниям, и 2 умерших младенцев.

Медико-генетическое консультирование (МГК) семей включало клинико-генеалогический анализ, синдромологическую диагностику с использованием компьютерных диагностических программ («London Medical Database», «СинДиаг»), оценку прогноза потомства.

\section{Результаты и обсуждение}

Проанализированы фенотипические данные 12 пациентов (2 живорожденных и 10 абортированных плодов) с клиническим диагнозом СФ. Соотношение лиц женского и мужского пола составило 10:2. Стандартный анализ кариотипа выполнен у 9 пробандов, хро- 
мосомных аномалий не выявлено (в 3 случаях родители отказались от исследования, но по клиническим и морфологическим данным фенотип плодов соответствовал СФ). Результаты пренатальной УЗ диагностики, клинического и патоморфологического исследований пациентов представлены в таблице, родословные отражены на рис. 1.

Наблюдение 1 (рис. 1, родословная I; таблица, случай 1). Семейный анамнез: брак не родственный, у 25-летней матери врожденный порок сердца (ВПС: ДМЖП), отец здоров.

Беременность пробандом первая. Пренатальный скрининг 1-2 триместров проводился по месту жительства семьи. В 11 недель гестации по данным УЗ исследования выявлена кистозная гигрома шеи (KTP - 50,0; Nt $-5,3)$. Супруги отказались от инвазивной диагностики и прерывания беременности. При обследовании плода в
23 недели гестации установлены комбинированный порок сердца (ДМПП, ДМЖП, перерыв нижней полой вены) и многоводие. Экспертная оценка состояния плода выполнена в «Республиканском научно-практическом центре «Мать и дитя» в 28 недель гестации. УЗ диагноз - МВПР: расщелина неба, правосторонняя ДГ, ВПС, гидронефроз (рис. 2). В связи с выраженным многоводием выполнено амниодренирование, образец околоплодных вод использован для цитогенетического исследования, кариотип плода - 46,XX.

В 33 недели беременности путем операции кесарево сечение родилась девочка с массой 1850 г, длиной тела 38 см, окружностью головы $31 \mathrm{cm.} \mathrm{Оценка} \mathrm{по} \mathrm{шка-}$ ле Апгар 5/ИВЛ. В связи с наличием пороков развития на 2 сутки жизни проконсультирована генетиком.

Фенотип пробанда (рис. 3) включал: ЧЛД (низкопосаженные дисморфичные ушные раковины, широ-

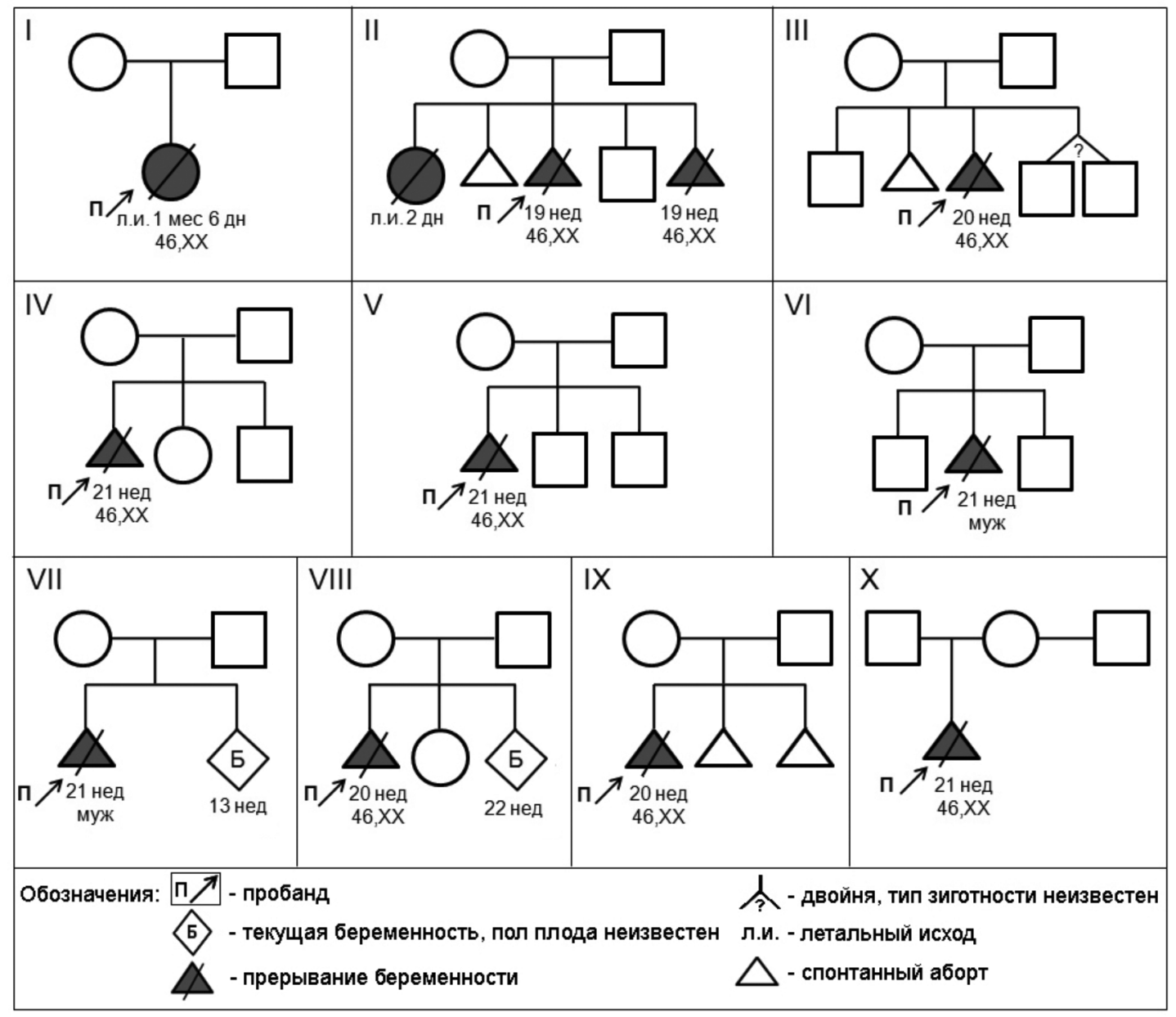

Рис. 1. Родословные семей, имеющих потомство с клиническим диагнозом СФ. 
кий лоб, капиллярные гемангиомы в области лба, переносья, век, широкое плоское переносье, гипертелоризм, монголоидный разрез глазных щелей, короткий нос с широкой спинкой и толстым кончиком, макростомия, микроретрогения), расщелину мягкого и твердого неба, короткую шею. При осмотре отмечены удлиненные первые пальцы (по данным рентгенографии исключен вариант 3-фаланговых пальцев) с проксимальной позицией и гипоплазией ногтевых пластинок, гипоплазия терминальных фаланг 2-4 пальцев, гипоплазия/аплазия ногтевых пластинок на 2-5 пальцах кистей; аплазия ногтевых пластинок 2-5 пальцев стоп. Диагностированы правосторонняя ДГ (пластика диафрагмы выполнена в возрасте 19 дней), ВПС: ДМПП,
ДМЖП, открытый аортальный проток (оперативное лечение проведено на 13 сутки жизни), гидронефроз. Клинический диагноз: СФ. В динамике у ребенка наблюдалась тяжелая задержка общего развития. Летальный исход в возрасте 46 суток. При аутопсии дополнительно выявлены аномалии головного мозга (внутренняя гидроцефалия, аринэнцефалия, нарушение цитоархитектоники коры больших полушарий), гипоплазия тонкой кишки, дивертикул Меккеля.

Наблюдение 2 (семейное; рис. 1, родословная II). Супруги здоровы, брак не родственный. Отягощенный репродуктивный анамнез.

Первая беременность: девочка родилась от срочных родов с массой 2570 г, длиной тела 47 см, окружностью

Фенотипические характеристики пациентов с синдромом Фринса

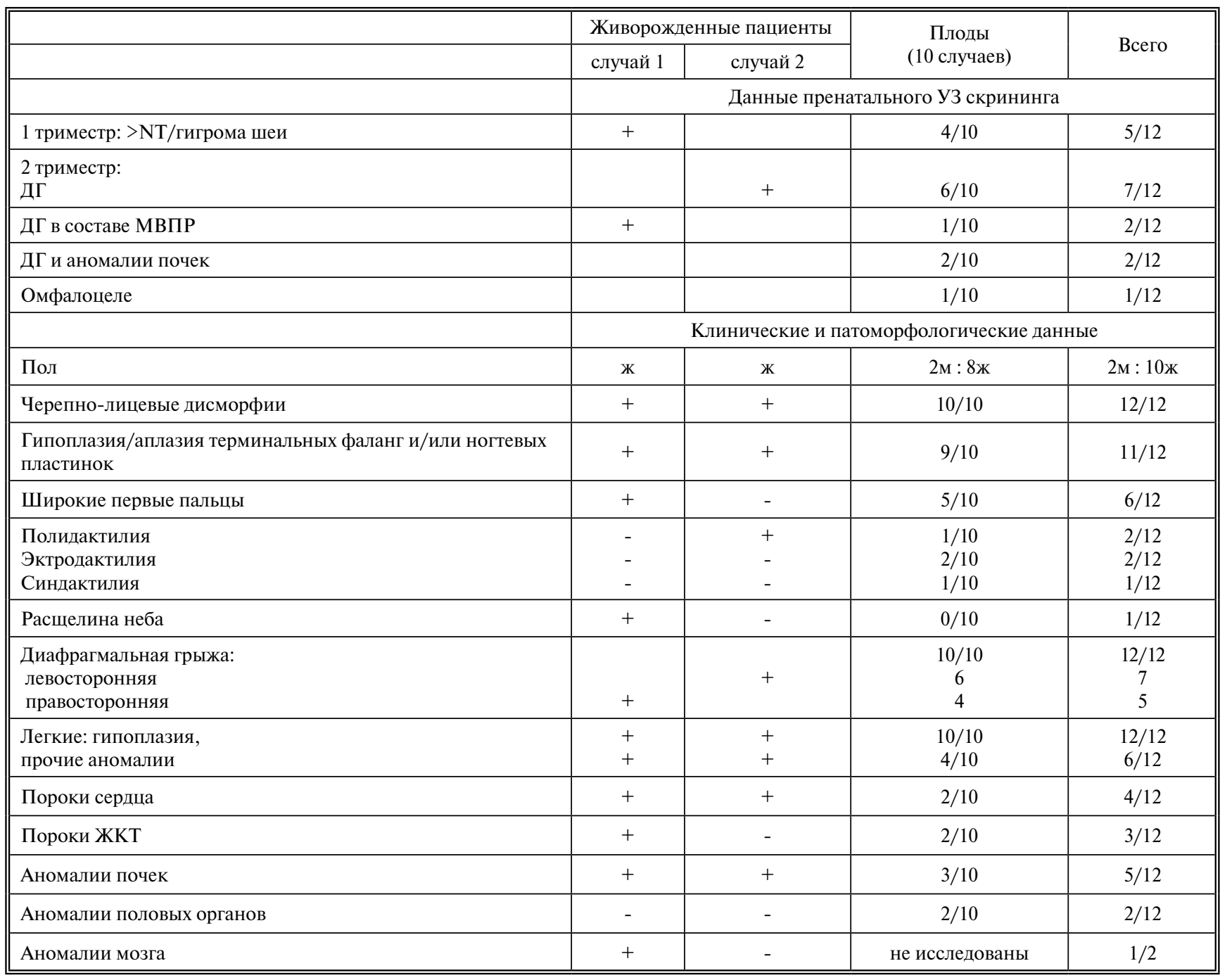


головы 31см, оценкой по шкале Апгар 1/ИВЛ. Фенотипические данные представлены в таблице (случай 2). При осмотре младенца были выявлены грубые черты лица, брахидактилия, аплазия терминальной фаланги 4 пальца левой кисти, аплазия ногтевых пластинок на пальцах правой кисти и левой стопы, полидактилия левой стопы, синдактилия 4-5 правой стопы. По данным рентгенографии идентифицирована левосторонняя ДГ. Летальный исход на 2 сутки жизни на фоне нарастающего метаболического ацидоза. При патоморфологическом исследовании подтверждена ДГ, установлены гипоплазия легких (масса 8 г при нормальном значении 40 г) с агенезией до- ли правого легкого, открытое овальное окно (ООО), умеренная гипоплазия правой почки, нефроптоз. Пороков развития мозга, ЖКТ и гениталий не выявлено. Клинический/патоморфологический диагноз: МВПР.

Вторая беременность неразвивающаяся в сроке 10 недель гестации.

Четвертая беременность закончилась рождением здорового мальчика.

При третьей и пятой беременностях по данным УЗ исследования в сроке гестации 11 недель отмечалось расширение воротникового пространства у плодов, у одного из них диагностированы гигрома шеи и начи-

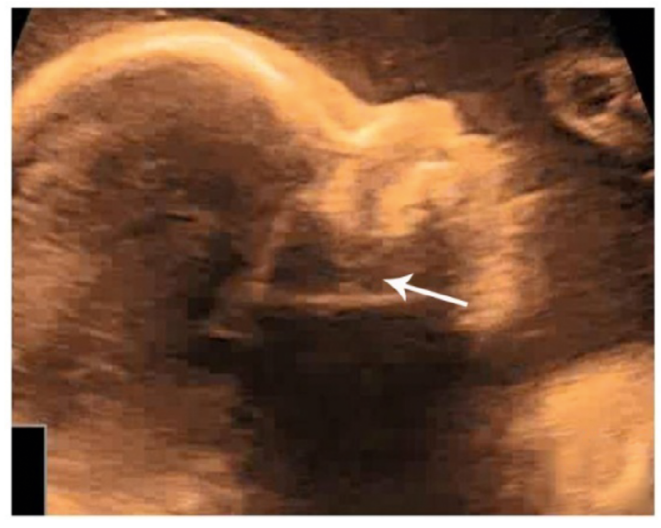

A

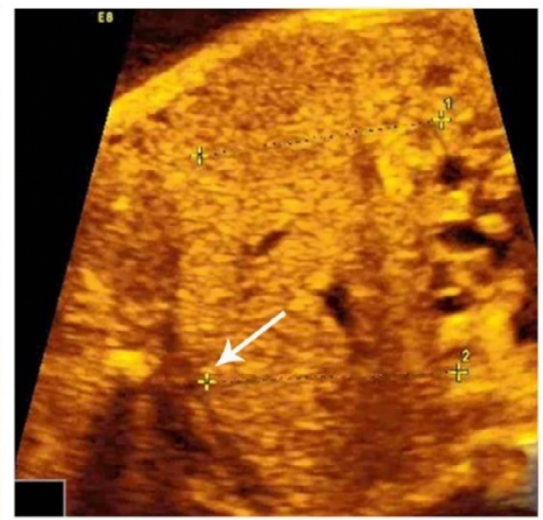

Б

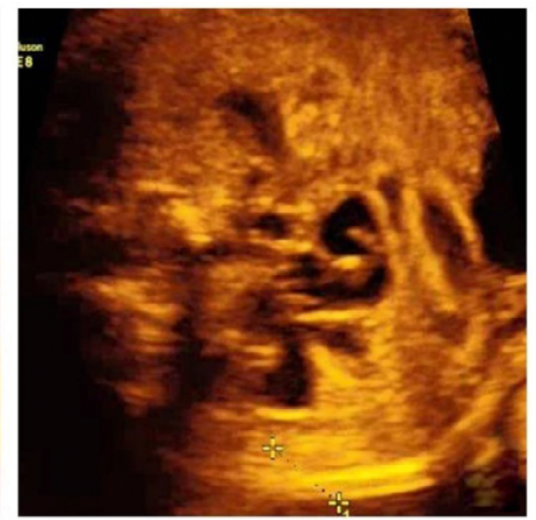

B

Рис. 2. Аномалии развития плода с СФ, установленные при пренатальном УЗ исследовании (А - расщелина неба, Б - ДГ, В - гидронефроз).

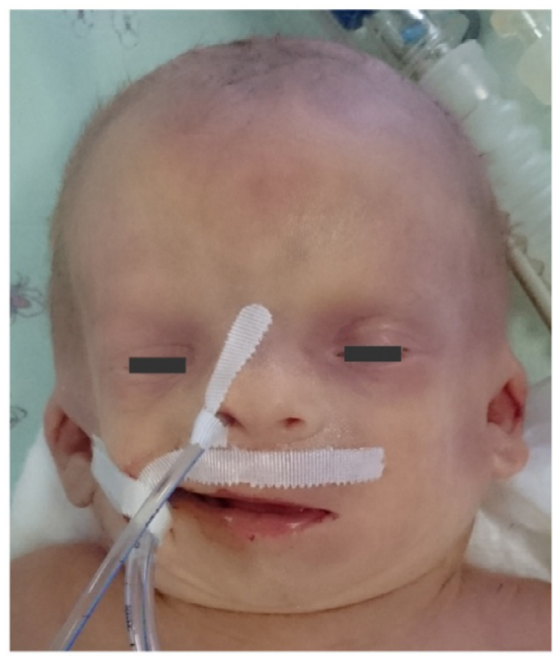

A
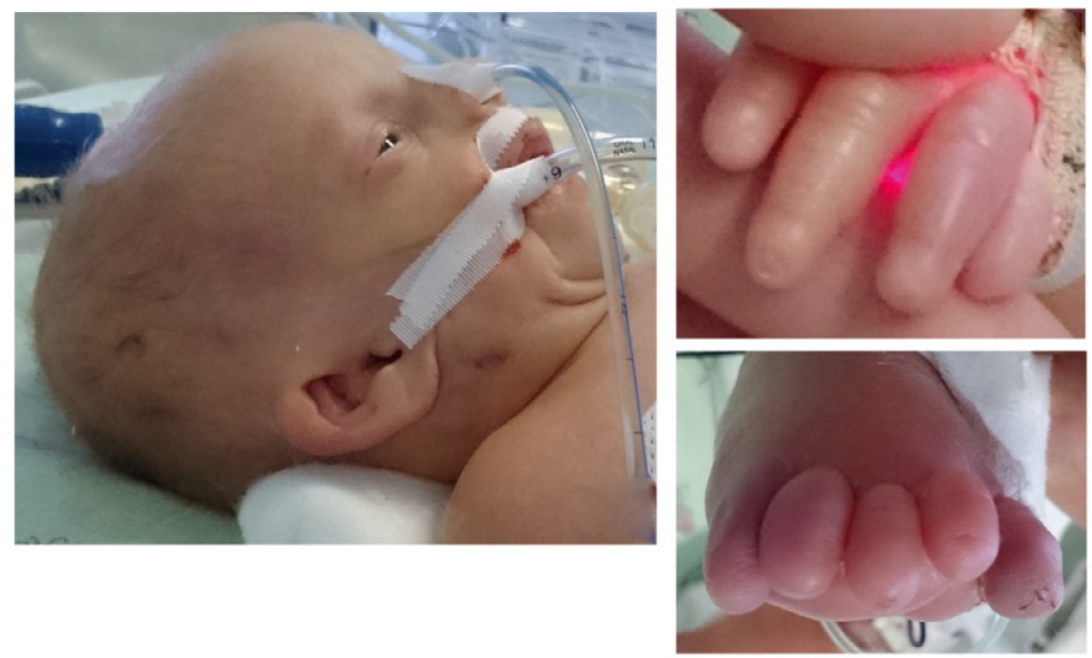

Б

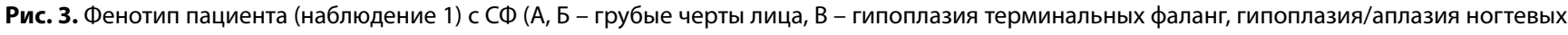
пластинок пальцев кистей, Г - аплазия ногтевых пластинок пальцев стоп). 
нающаяся водянка. В 17 и 19 недель у плодов выявлена ДГ. По результатам пренатального цитогенетического анализа определены нормальные кариотипы $(46, X X)$. При пятой беременности на основании семейного анамнеза и наблюдаемых характерных УЗ данных был установлен пренатальный диагноз СФ. Беременности прерваны по медицинским показаниям. Подробное описание результатов патоморфологического исследования обоих плодов в данной семье ранее опубликовано Новиковой И.В. и соавт. [22]. При осмотре отмечены лицевые дисморфии, гипоплазия терминальных фаланг/ногтевых пластинок, у одного из плодов сочетание преаксиальной полидактилии кистей с эктродактилией стоп, у второго - эктродактилия левой стопы. В обоих случаях подтверждено наличие ДГ, выявлена гипоплазия легких и наружных гениталий. У одного из плодов установлен ВПС (общий артериальный ствол), L-образная почка, двурогая матка. Патоморфологический диагноз: СФ.

В семье прослеживается аутосомно-рецессивный тип наследования комплекса МВПР и сходство сибсов (3/3) по ведущим признакам СФ (ДГ, гипоплазии легких, ЧЛД, гипоплазии терминальных фаланг/ногтевых пластинок) и редким аномалиям конечностей: полидактилии $(2 / 3)$ и эктродактилии $(2 / 3)$. Заключительный диагноз у потомства - СФ.

Плоды с СФ (рис. 1, родословные II-X). Обобщенные УЗ и патоморфологические данные 10 плодов (включая 3 ранее описанных изолированных случая [23] и плоды, относящиеся к семейному наблюдению) представлены в таблице. Во всех семьях беременность была прервана по медицинским показаниям в 19-21 неделю гестации, диагноз СФ установлен по результатам патоморфологического исследования. Фенотипические проявления СФ у плода отражены на рис. 4.

Пренатальные УЗ признаки СФ. При проведении пренатальной УЗ диагностики у 5 пациентов исследуемой группы имело место расширение воротникового пространства, во 2 триместре у 11 из 12 плодов была выявлена ДГ, в 4 случаях ассоциированная с другими аномалиями (расщелиной неба, пороком сердца, гиперэхогенными почками, гидронефрозом). В одном наблюдении первично диагностированным пороком развития в 19 недель гестации было омфалоцеле, впоследствии при аутопсии плода дополнительно обнаружена ДГ. Несмотря на раннее выявление УЗ маркеров, соответствующих проявлениям СФ, синдромальный диагноз в изолированных случаях не был установлен пренатально.

По опубликованным данным, спектр УЗ признаков СФ зависит от срока гестации: в 1 триместре были отмечены расширение воротникового пространства/ гигрома шеи, водянка плода [7-9, 16, 24], во 2 триместре выявлены ДГ (ключевой маркер СФ) и многоводие [3, 4, 6-9, 15-21, 24, 25]. Среди ассоциированных с ДГ аномалий развития описаны орофациальные рас-

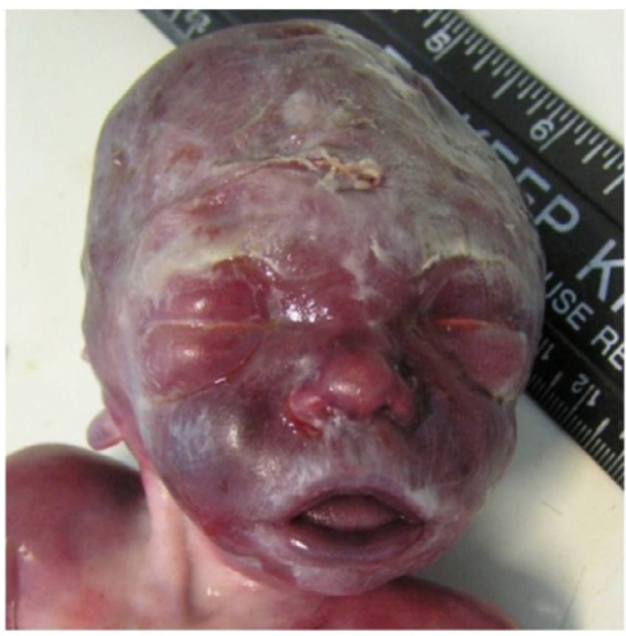

A

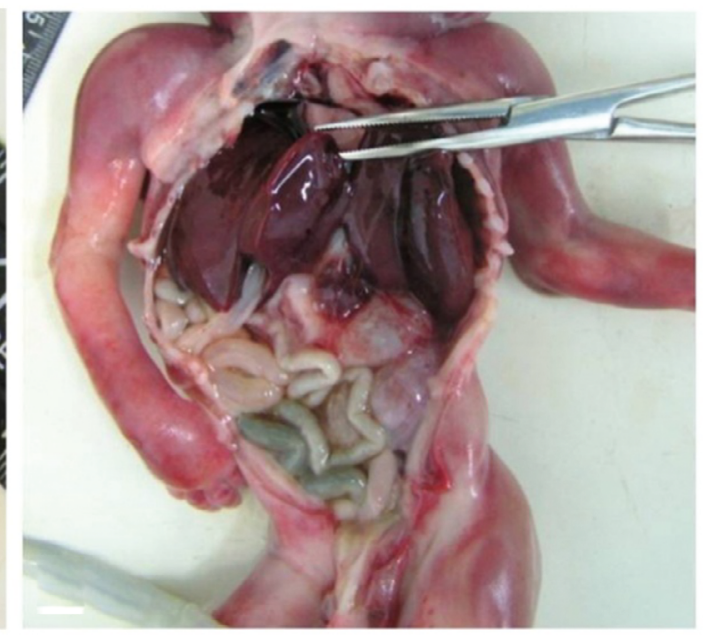

Б

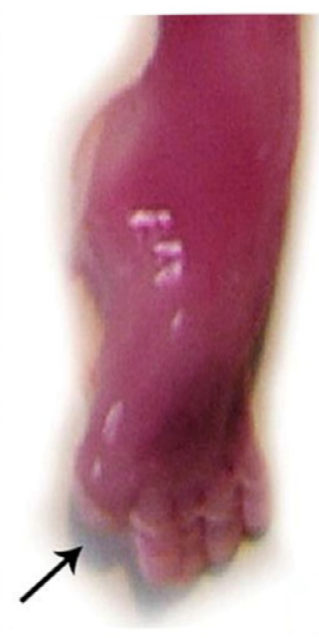

B

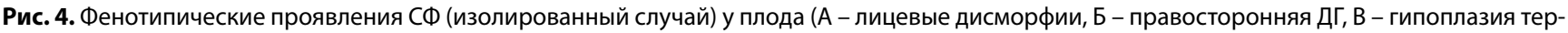
минальной фаланги 1 пальца стопы). 
щелины [7-10], аномалии мозга $[9,10,15,21]$, пороки сердца $[8,9]$ и почек $[5,8,21]$, а также омфалоцеле [7, 9]. Во всех вышеприведенных наблюдениях, а также в последнем опубликованном в 2018 г. случае [26] СФ по УЗ данным заподозрен не был, диагноз установлен после клинического осмотра и/или патоморфологического исследования. Необходимость постнатального обследования плода/мертворожденного подчеркивают Peron А. и соавт. [21], представившие в 2014 г. преи постнатальные характеристики 5 пациентов с СФ.

В то же время, описаны семьи (мы располагаем информацией о 12 наблюдениях), в которых диагноз СФ у плода с ДГ и пороками развития был первично сформулирован пренатально на основании семейного анамнеза (наличие больных сибсов) $[2,7,13]$ либо характерных УЗ данных [10, 27-34]. Использование режима объемной реконструкции (3D) и магнитно-резонансной томографии (МРТ) позволило уточнить наличие у плода ассоциированных с ДГ аномалий развития, характерных для СФ: грубых черт лица [28, 34], расщелин губы, неба, их сочетания [10, 31, 32], порока Дэнди-Уокера, агенезии мозолистого тела, гидроцефалии [10, 30, 34], пороков сердца [29, 30, 34] и почек (кисты, гиперэхогенные почки, пиелоэктазии) [10, 13, $29,31,33]$, а также более редких врожденных нарушений - омфалоцеле [10], аномалий кистей [29]. У3 диагноз СФ был подтвержден результатами осмотра и аутопсии абортированных плодов/умерших младенцев.

Фенотипические проявления у представленных нами пациентов соответствуют диагностическим критериям СФ. Так, грубые черты лица были отмечены как у живорожденных, так и у плодов. ДГ с преобладанием левостороннего поражения (7/12) и гипоплазия легких были установлены у всех 12 пациентов. Редкие аномалии развития легких представлены кистозно-аденоматозным пороком (2/12), легочным секвестром (2/12) и аплазией доли легкого (1/12). Характерные дефекты терминальных фаланг и/или ногтевых пластинок отмечались в 11 из 12 наблюдений.

Спектр ассоциированных пороков в исследуемой группе также не отличается от опубликованных данных. Расщелина неба и патология головного мозга (аринэнцефалия и гидроцефалия) отмечены у одного из двух живорожденных пациентов. В трех случаях выявлен комбинированный ВПС (ДМПП, ДМЖП, ОАП; ДМЖП и двустворчатый аортальный клапан; общий артериальный ствол). Патология ЖКТ представлена омфалоцеле (1 случай) и дивертикулом Меккеля (у 2 пациентов), аномалии мочеполовой системы - гидронефрозом (2 случая) и единичными наблюдениями подковообразной почки, гипоплазии и кистозной дисплазии почек, двурогой матки и гипоспадии.
В соответствии с международными критериями [12] 9 пациентов (изолированные случаи СФ) могут быть отнесены к первой категории.

Анализ проявлений у сибсов имеет большое значение в оценке фенотипической вариабельности СФ. Опубликованные семейные наблюдения иллюстрируют высокую степень внутрисемейного сходства по ведущим клиническим признакам (ДГ, гипоплазии фаланг/ногтевых пластинок), орофациальным расщелинам и порокам сердца. В то же время, описаны сибсы, в том числе монозиготные близнецы, дискордантные по наличию ДГ [5, 7, 15, 16, 18]. Вариабельность проявлений прослеживается в отношении пороков мозга $[5,16]$, зрительного анализатора $[6,8,9,14,15]$, омфалоцеле [8, 18], аномалий кишечника [3, 14], селезенки $[13,14]$ и почек $[5,14]$.

В представленном семейном наблюдении все сибсы (3/3) имели ДГ и гипоплазию легких, тогда как среди ассоциированных аномалий развития тяжелые формы пороков (общий артериальный ствол, L-образная почка, двурогая матка) были выявлены только у одного плода.

У 3 сибсов отмечено сочетание типичных для СФ аномалий развития конечностей (гипоплазия терминальных фаланг и ногтевых пластинок) с редкими (полидактилия кистей, эктродактилия стоп). Сравнительный анализ фенотипов СФ и синдромов, манифестирующих эктродактилией/полидактилией (эКтродактилии-полидактилии, Ectrodactyly-polydactyly, ОМІМ 225290; эктродактилии, эктодермальной дисплазии и расщелины губы/неба 1 и 2 типов, EЕC1, OMIM \%129900; EEC2, ОМIM \#604292; эктодермальной дисплазии, эктродактилии и макулярной дистрофии, EEMS, OMIM \#225280; брахидактилии-эктродактилии с аплазией/гипоплазией малоберцовой кости, Brachydactyly-ectrodactyly with fibular aplasia or hypoplasia, OMIM 113310; эктродактилии-расщелины неба, ЕСР, OMIM 129830; Паттерсона-Стивенсона-Фонтейна, Patterson-Stevenson-Fontaine syndrome, OMIM 183700; Гершон-Баруха, Gershoni-Baruch syndrome, OMIM 609545), показал различия по диагностически значимым симптомам или по типу наследования, т.е. оснований для изменения диагноза СФ у потомства в данной семье нет.

Поскольку описания пациентов с СФ, имеющих ассоциацию ДГ с полидактилией/ олигодактилией/ эктродактилией, исключительно редки (по классификации A. Lin и соавт. [12] относятся к третьей категории - атипичный СФ), оценка диагностической значимости данных пороков конечностей представляет особый интерес.

Ramsing M. с соавт. [14] опубликовали 2 семейных наблюдения СФ (5 пациентов с МВПР) с данными пато- 
морфологического исследования. В одной семье с 3 пораженными сибсами умерший младенец имел постаксиальную полидактилию кистей и синдактилию стоп, тогда как у двух плодов данные варианты пороков отсутствовали. В другой семье у одного плода выявлена синдактилия кистей/стоп, у второго - олигосиндактилия стоп [14]. Преаксиальная полидактилия кисти была описана у младенца с нормальным кариотипом и клиническими проявлениями, соответствующими фенотипу СФ, которые были оценены авторами как «Fryns-like phenotype» [35].

Также известно наблюдение плода с МВПР в кровнородственном браке с исключенным хромосомным дисбалансом и мутациями в гене $P I G N$, у которого клинический симптомокомплекс соответствовал фенотипу СФ и была отмечена олигодактилия стопы - два рудиментарных пальца с отсутствием ногтевых пластинок [36].

Первое наблюдение эктродактилии (обозначенной авторами как «three digit ectrodactyly»), ассоциированной с симптомокомплексом СФ, было приведено Sheffield J.S. с соавт.: правая кисть плода была представлена двухфаланговым пальцем, расположенным с радиальной стороны, центральным трехфаланговым пальцем и рудиментарным двухфаланговым пальцем с аплазией ногтевой пластинки с ульнарной стороны [29]. Кроме того, в 1995 г. Saal H. и Bulas D. описали новорожденного с комплексом МВПР (ДГ, агенезия мозолистого тела, ДМЖП, эктродактилия) и нормальным кариотипом, оценив его как уникальный случай. Девочка умерла в первые сутки жизни, диагноз СФ не был рассмотрен авторами [37]. Анализируя данное сообщение, можно отметить, что в настоящее время ДМЖП и агенезия мозолистого тела, выявленные у младенца, включены в фенотипический спектр СФ, что позволяет рассматривать этот случай как первое описание СФ с эктродактилией.

Таким образом, представленные нами пациенты дополняют наблюдение Sheffield J.S. с соавт. [29], а редкие аномалии кистей и стоп - пре/постаксиальная полидактилия, олигодактилия, эктродактилия, их сочетание, могут рассматриваться в качестве проявлений СФ.

Дифференциальная диагностика. Поскольку ДГ является ключевым признаком СФ, были рассмотрены моногенные синдромы МВПР с высокой частотой встречаемости данного порока: Симпсона-Голаби-Бэмеля (Simpson-Golabi-Behmel, OMIM \#312870); ДоннаиБэрроy (Donnai-Barrow, OMIM \#222448); Мэтью-Вуда (Matthew-Wood, OMIM \#601186); Гершон-Баруха (Gershoni-Baruch, ОМІМ 609545)). В отличие от СФ, эти заболевания не относятся к летальным синдромам, некоторые отличаются типом наследования.
Клинические признаки, соответствующие проявлениям СФ, также описаны при ряде известных хромосомных синдромов (Палистера-Киллиана, трисомии 18 и 22) и различных формах структурного хромосомного дисбаланса (дупликация 1q22-q32, изохромосома 6q, делеция 8p23, делеция 15q26, кольцевая хромосома 15, микроделеция 15q24, микроделеция/дупликация $16 \mathrm{p} 11.2$ и др.). В связи с этим, клинический диагноз СФ может рассматриваться только после исключения хромосомной патологии.

Репродуктивный анамнез семей. Результат анализа исходов беременностей в представленных семьях (рис. 1) свидетельствует об отягощенном акушерском анамнезе. Из 29 беременностей в 12 случаях потомство имело СФ. Четыре беременности завершились спонтанным абортом в 1 триместре, 11 - рождением здоровых детей, в 2 семьях при текущих беременностях ВПР плода по данным УЗ скрининга не выявлено. Полученные данные согласуются с опубликованными наблюдениями по патологии репродукции в семьях, отягощенных по СФ.

\section{Заключение}

Клинический диагноз СФ устанавливается при соответствии комплекса МВПР принятым диагностическим критериям после исключения хромосомного дисбаланса с использованием цитогенетических, молекулярно-цитогенетических и молекулярно-генетических методов.

Ведушим диагностическим признаком СФ является ДГ. При обнаружении у плода данного порока развития следует проводить целенаправленный поиск ассоциированных аномалий с использованием возможностей режима $3 \mathrm{D}$ и MPT.

На основании опубликованных и представленных наблюдений полидактилия и эктродактилия могут рассматриваться в качестве элементов фенотипического спектра СФ.

\section{Список литературы}

1. Fryns J.P., Moerman F., Goddeeris P. et al. A new lethal syndrome with cloudy cornea, diaphragmatic defects and distal limb deformities. Hum. Genet. 1979; 50(1): 65-70.

2. Ayme S., Julian C., Gambarelli D. et al. Fryns Syndrome: report on 8 new cases. Clin. Genet. 1989; 35(3): 191-201.

3. Schwyzer U., Briner J., Schinzel A. Fryns syndrome in a girl born to consanguineous parents. Acta. Paediatr. Scand. 1987; 76(1): 167-171.

4. Roy M., Chikkannaiah P., Bali A. Congenital diaphragmatic hernia with hypoplastic lungs, heart, and additional anomalies: A case of? Fryns syndrome. J. NTR. Univ. Health. Sci. 2013; 2(2): 130-132.

5. Lubinsky M., Severn C., Rapoport J.M. Fryns Syndrome: A new variable multiple congenital anomaly (MCA) syndrome. Am. J. Med. Genet. 1983; 14(3): 461-466. 
6. Moerman P., Fryns J.P., Vandenberghe K. et al. The syndrome of diaphragmatic hernia, abnormal face and distal limb anomalies (Fryns syndrome): report of two sibs with further delineation of this multiple congenital anomaly (MCA) syndrome. Am. J. Med. Genet. 1988; 31(4): 805-814.

7. Bamforth J.S., Leonard C.O., Chodirker B.N. et al. Congenital diaphragmatic hernia, coarse facies, and acral hypoplasia: Fryns syndrome. Am. J. Med. Genet. 1989; 32(1): 93--99.

8. Alessandri J.L., Cuillier F., Malan V. et al. Fryns syndrome without diaphragmatic hernia, DOOR syndrome or Fryns-like syndrome? Report on patients from Indian Ocean islands. Am. J. Med. Genet. 2014; 164A(3): 648-654.

9. Alessandri J.L., Gordon C.T., Jacquemont M.L. et al. Recessive loss of function PIGN alleles, including an intragenic deletion with Fryns syndrome. Eur. J. Hum. Genet. 2018; 26(3): 340-349.

10. McInerney-Leo A.M., Harris J.E., Gattas M. et al. Fryns syndrome associated with recessive mutations in PIGN in two separate families. Hum. Mutat. 2016; 37(7): 695-702.

11. Slavotinek A.M. Fryns Syndrome: a review of the phenotype and diagnostic guidelines. Am. J. Med. Genet. 2004; 124A(4): 427-433.

12. Lin A.E., Pober B.R., Mullen M.P., Slavotinek A.M. Cardiovascular malformations in Fryns syndrome: is there a pathogenic role for neural crest cells? Am. J. Med. Genet. 2005; 139(3): 186-193.

13. Pellissier M.C., Philip N., Potier A. et al. Prenatal diagnosis of Fryns' syndrome. Prenat. Diagn. 1992; 12(4): 299-303.

14. Ramsing M., Gillesen-Kaesbach G., Holzgreve W. et al. Variability in the phenotypic expression of Fryns syndrome: a report of two sibships. Am. J. Med. Genet. 2000; 95(5): 415-424.

15. Vargas J.E., Cox G.F., Korf B.R. Discordant phenotype in monozygotic twins with Fryns syndrome. Am. J. Med. Genet. 2000; 94(1): 42-45.

16. Pratap A., Agrawal A., Raja S. et al. Fryns syndrome: a lethal mesoectodermal birth defect with variable expression in a pair of monozygotic twins. Singapore Med. J. 2007; 48(4): 106.

17. Nirmaladevi M., Kurup S., Ajitha E.V. Fryns syndrome in monozygotic twins - a case report with review of literature. Int. J. Morphol. 2012; 30(1): 56-60.

18. Arora K., Thukral A., Das R.R. et al. Fryns syndrome: a lethal birth defect with variable phenotypic expressions in siblings. Indian J. Pediatr. 2014; 81(6): 614-616.

19. Samueloff A., Navot D., Birkenfeld A., Schenker J.G. Fryns syndrome: a predictable, lethal pattern of multiple congenital anomalies. Am. J. Obstet. Gynecol. 1987; 156(1): 86-88.

20. Dentici M.L., Brancati F., Mingarelli R., Dallapiccola B. A 6-years old child with Fryns syndrome: further delineation of the natural history of the condition in survivors. Eur. J. Med. Genet. 2009; 52(6): 421-425.

21. Peron A., Bedeschi M.F., Fabietti I. et al. Prenatal and postnatal findings in five cases of Fryns syndrome. Prenat. Diagns. 2014; 34(12): $1227-1230$

22. Новикова И.В., Ковалев С.И., Мараховская Э.И. Семейный случай синдрома Фринса у двух плодов II триместра. Пренатальная диагностика. 2018; 17(2): 164-169.

23. Ильина Е.Г., Новикова И.В., Ершова А.А. и др. Три случая синдрома Фринса, диагностированных пренатально в городе Минске за один месяц. Медииинская генетика. 2010; (5): 44-47.

24. Bulas D.I., Saal H.M., Allen J.F. et al. Cystic hygroma and congenital diaphragmatic hernia: early prenatal sonographic evaluation of Fryns syndrome. Prenat. Diagn. 1992; 12(11): 867-875.

25. Arakeri S.U., Manchanda J. Congenital diaphragmatic hernia associated with Fryns syndrome - an autopsy study. J. Evol. Med. Dent. Scienc. 2012; 1(5): 703-706.

26. Dwivedi T., Hungund B. Fryns syndrome: a rare case report with review of literature. J. NTR. Univ. Health Sci. 2018; 7(2): 147-153.
27. Manouvrier-Hanu S., Devisme L., Vaast P. et al. Fryns syndrome and erupted teeth in a 24-week-old fetus. Genet. Couns. 1996; 7(2): $131-134$.

28. Wymersch D.V., Favre R., Gasser B. Use of three-dimensional ultrasound to establish the prenatal diagnosis of Fryns syndrome. $\mathrm{Fe}$ tal. Diagn. Ther. 1996; 11(5): 335-340.

29. Sheffield J.S., Twickler D.M., Timmons C. et al. Fryns syndrome: prenatal diagnosis and pathologic correlation. J. Ultrasound. Med. 1998; 17(9): 585-589.

30. Saliani P., Epstein S., Cohen D. The role of ultrasound in the diagnosis of Fryns syndrome. J. Diagn. Med. Sonogr. 2004; 20(1): 42-45.

31. Benacerraf B.R., Sadow P.M., Barnewolt C.E. et al. Cleft of the secondary palate without cleft lip diagnosed with three-dimensional ultrasound and magnetic resonance imaging in a fetus with Fryns' syndrome. Ultrasound Obste.t Gynecol. 2006; 27(5): 566-570.

32. Yucesoy G., Cakiroglu Y., Caliskan E. Fryns syndrome: case report and review of the literature. J. Clin. Ultrasound. 2008; 36(5): 315-317.

33. Tosun M., Celik H., Bese E. et al. Prenatal diagnosis of Fryns syndrome: a case report. Gynecol. Obstet. Reprod. Med. 2009; 15(1): 47-49.

34. Плотко И.С., Федотов В.П., Соболева И.А., Машнев Е.Ю. Пренатальная диагностика редких врожденных пороков и синдромов. XXXII. Синдром Фринса. Пренатальная диагностика. 2009; 8(2): $112-114$

35. Tatar A., Öztas S., Örs R. A case with Fryns syndrome-like phenotype. $A \ddot{U} T D .2005 ; 37: 19-21$.

36. Brady P.D., Moerman P., De Catte L. et al. Exome sequencing identifies a recessive $P I N G$ splice mutation as a cause of syndromic congenital diaphragmatic hernia. Eur. J. Med. Genet. 2014; 57(9): 487493.

37. Saal H.M., Bulas D.I. Ectrodactyly, diaphragmatic hernia, congenital heart defect, and agenesis of the corpus callosum. Clinl. Dysmorphol. 1995; 4(3): 246-250.

\section{References}

1. Fryns J.P., Moerman F., Goddeeris P. et al. A new lethal syndrome with cloudy cornea, diaphragmatic defects and distal limb deformities. Hum. Genet. 1979; 50(1): 65-70.

2. Ayme S., Julian C., Gambarelli D. et al. Fryns Syndrome: report on 8 new cases. Clin. Genet. 1989; 35(3): 191-201.

3. Schwyzer U., Briner J., Schinzel A. Fryns syndrome in a girl born to consanguineous parents. Acta. Paediatr. Scand. 1987; 76(1): 167171.

4. Roy M., Chikkannaiah P., Bali A. Congenital diaphragmatic hernia with hypoplastic lungs, heart, and additional anomalies: A case of? Fryns syndrome. J. NTR. Univ. Health. Sci. 2013; 2(2): 130-132.

5. Lubinsky M., Severn C., Rapoport J.M. Fryns Syndrome: A new variable multiple congenital anomaly (MCA) syndrome. Am. J. Med. Genet. 1983; 14(3): 461-466.

6. Moerman P., Fryns J.P., Vandenberghe K. et al. The syndrome of diaphragmatic hernia, abnormal face and distal limb anomalies (Fryns syndrome): report of two sibs with further delineation of this multiple congenital anomaly (MCA) syndrome. Am. J. Med. Genet. 1988; 31(4): 805-814.

7. Bamforth J.S., Leonard C.O., Chodirker B.N. et al. Congenital diaphragmatic hernia, coarse facies, and acral hypoplasia: Fryns syndrome. Am. J. Med. Genet. 1989; 32(1): 93--99.

8. Alessandri J.L., Cuillier F., Malan V. et al. Fryns syndrome without diaphragmatic hernia, DOOR syndrome or Fryns-like syndrome? Report on patients from Indian Ocean islands. Am. J. Med. Genet. 2014; 164A(3): 648-654.

9. Alessandri J.L., Gordon C.T., Jacquemont M.L. et al. Recessive loss of function PIGN alleles, including an intragenic deletion with Fryns syndrome. Eur. J. Hum. Genet. 2018; 26(3): 340-349. 
10. McInerney-Leo A.M., Harris J.E., Gattas M. et al. Fryns syndrome associated with recessive mutations in PIGN in two separate families. Hum. Mutat. 2016; 37(7): 695-702.

11. Slavotinek A.M. Fryns Syndrome: a review of the phenotype and diagnostic guidelines. Am. J. Med. Genet. 2004; 124A(4): 427-433.

12. Lin A.E., Pober B.R., Mullen M.P., Slavotinek A.M. Cardiovascular malformations in Fryns syndrome: is there a pathogenic role for neural crest cells? Am. J. Med. Genet. 2005; 139(3): 186-193.

13. Pellissier M.C., Philip N., Potier A. et al. Prenatal diagnosis of Fryns' syndrome. Prenat. Diagn. 1992; 12(4): 299-303.

14. Ramsing M., Gillesen-Kaesbach G., Holzgreve W. et al. Variability in the phenotypic expression of Fryns syndrome: a report of two sibships. Am. J. Med. Genet. 2000; 95(5): 415-424.

15. Vargas J.E., Cox G.F., Korf B.R. Discordant phenotype in monozygotic twins with Fryns syndrome. Am. J. Med. Genet. 2000; 94(1): 42-45.

16. Pratap A., Agrawal A., Raja S. et al. Fryns syndrome: a lethal mesoectodermal birth defect with variable expression in a pair of monozygotic twins. Singapore Med. J. 2007; 48(4): 106.

17. Nirmaladevi M., Kurup S., Ajitha E.V. Fryns syndrome in monozygotic twins - a case report with review of literature. Int. J. Morphol. 2012; 30(1): 56-60.

18. Arora K., Thukral A., Das R.R. et al. Fryns syndrome: a lethal birth defect with variable phenotypic expressions in siblings. Indian J. Pediatr. 2014; 81(6): 614-616.

19. Samueloff A., Navot D., Birkenfeld A., Schenker J.G. Fryns syndrome: a predictable, lethal pattern of multiple congenital anomalies. Am. J. Obstet. Gynecol. 1987; 156(1): 86-88.

20. Dentici M.L., Brancati F., Mingarelli R., Dallapiccola B. A 6-years old child with Fryns syndrome: further delineation of the natural history of the condition in survivors. Eur. J. Med. Genet. 2009; 52(6): 421-425.

21. Peron A., Bedeschi M.F., Fabietti I. et al. Prenatal and postnatal findings in five cases of Fryns syndrome. Prenat. Diagns. 2014; 34(12): $1227-1230$

22. Novikova I.V., Kovalev S.I., Marahovskaya E.I. Semejnyj sluchaj sindroma Frinsa u dvuh plodov II trimestra. [Familial case of Fryns syndrome in two fetuses in second trimester]. Prenatal'naya diagnostika [Prenatal diagnostics] 2018; 17(2): 164-169. (In Russ.)

23. Il'ina E.G., Novikova I.V., Ershova A.A. i dr. Tri sluchaya sindroma Frinsa, diagnostirovannyh prenatal'no v gorode Minske za odin mesyac. [Three cases of Fryns syndrome prenatally diagnosed in
Minsk during one month]. Medicinskaya genetika [Medical genetics] 2010; (5): 44-47. (In Russ.)

24. Bulas D.I., Saal H.M., Allen J.F. et al. Cystic hygroma and congenital diaphragmatic hernia: early prenatal sonographic evaluation of Fryns syndrome. Prenat. Diagn. 1992; 12(11): 867-875.

25. Arakeri S.U., Manchanda J. Congenital diaphragmatic hernia associated with Fryns syndrome - an autopsy study. J. Evol. Med. Dent. Scienc. 2012; 1(5): 703-706.

26. Dwivedi T., Hungund B. Fryns syndrome: a rare case report with review of literature. J. NTR. Univ. Health Sci. 2018; 7(2): 147-153.

27. Manouvrier-Hanu S., Devisme L., Vaast P. et al. Fryns syndrome and erupted teeth in a 24-week-old fetus. Genet. Couns. 1996; 7(2): 131-134.

28. Wymersch D.V., Favre R., Gasser B. Use of three-dimensional ultrasound to establish the prenatal diagnosis of Fryns syndrome. Fetal. Diagn. Ther. 1996; 11(5): 335-340.

29. Sheffield J.S., Twickler D.M., Timmons C. et al. Fryns syndrome: prenatal diagnosis and pathologic correlation. J. Ultrasound. Med. 1998; 17(9): 585-589.

30. Saliani P., Epstein S., Cohen D. The role of ultrasound in the diagnosis of Fryns syndrome. J. Diagn. Med. Sonogr. 2004; 20(1): 42-45.

31. Benacerraf B.R., Sadow P.M., Barnewolt C.E. et al. Cleft of the secondary palate without cleft lip diagnosed with three-dimensional ultrasound and magnetic resonance imaging in a fetus with Fryns' syndrome. Ultrasound Obste.t Gynecol. 2006; 27(5): 566-570.

32. Yucesoy G., Cakiroglu Y., Caliskan E. Fryns syndrome: case report and review of the literature. J. Clin. Ultrasound. 2008; 36(5): 315-317.

33. Tosun M., Celik H., Bese E. et al. Prenatal diagnosis of Fryns syndrome: a case report. Gynecol. Obstet. Reprod. Med. 2009; 15(1): 47-49.

34. Plotko I.S., Fedotov V.P., Soboleva I.A., Mashnev E.Yu. Pre natal'naya diagnostika redkikh vrozhdennykh porokov i sindromov. XXXII. Sindrom Frinsa. [Prenatal diagnosis of rare congenital malformations and mov. XXXII. Frins syndrome. Prenatal'naya diagnostika [Prenatal diagnosis]. 2009; 8 (2): 112-114 (In Russ.)

35. Tatar A., Öztas S., Örs R. A case with Fryns syndrome-like phenotype. $A \ddot{U} T D .2005 ; 37:$ 19-21.

36. Brady P.D., Moerman P., De Catte L. et al. Exome sequencing identifies a recessive $P I N G$ splice mutation as a cause of syndromic congenital diaphragmatic hernia. Eur. J. Med. Genet. 2014; 57(9): 487-493.

37. Saal H.M., Bulas D.I. Ectrodactyly, diaphragmatic hernia, congenital heart defect, and agenesis of the corpus callosum. Clinl. Dysmorphol. 1995; 4(3): 246-250. 